\title{
Radiative Relaxation in Luminescent Silicon Nanocrystal Thiol-Ene Composites
}

\author{
Mahmud Sefannaser, ${ }^{\dagger}$ Salim A. Thomas,${ }^{\S}$ Kenneth J. Anderson, ${ }^{\diamond}$ Reed J. Petersen, ${ }^{\dagger}$ \\ Samuel L. Brown, ${ }^{\S}$ Philip Raymond Boudjouk, ${ }^{\diamond}$ Todd A. Pringle ${ }^{\S}$ Erik K. Hobbie ${ }^{* \dagger, \S, \beta}$ \\ ${ }^{\dagger}$ Department of Physics, North Dakota State University, Fargo, North Dakota 58108 \\ ${ }^{\S}$ Materials \& Nanotechnology Program, North Dakota State University, Fargo, North
} Dakota 58108

${ }^{\diamond}$ Department of Chemistry \& Biochemistry, North Dakota State University, Fargo, North Dakota 58108

${ }^{\beta}$ Department of Coatings \& Polymeric Materials, North Dakota State University, Fargo, North Dakota 58108

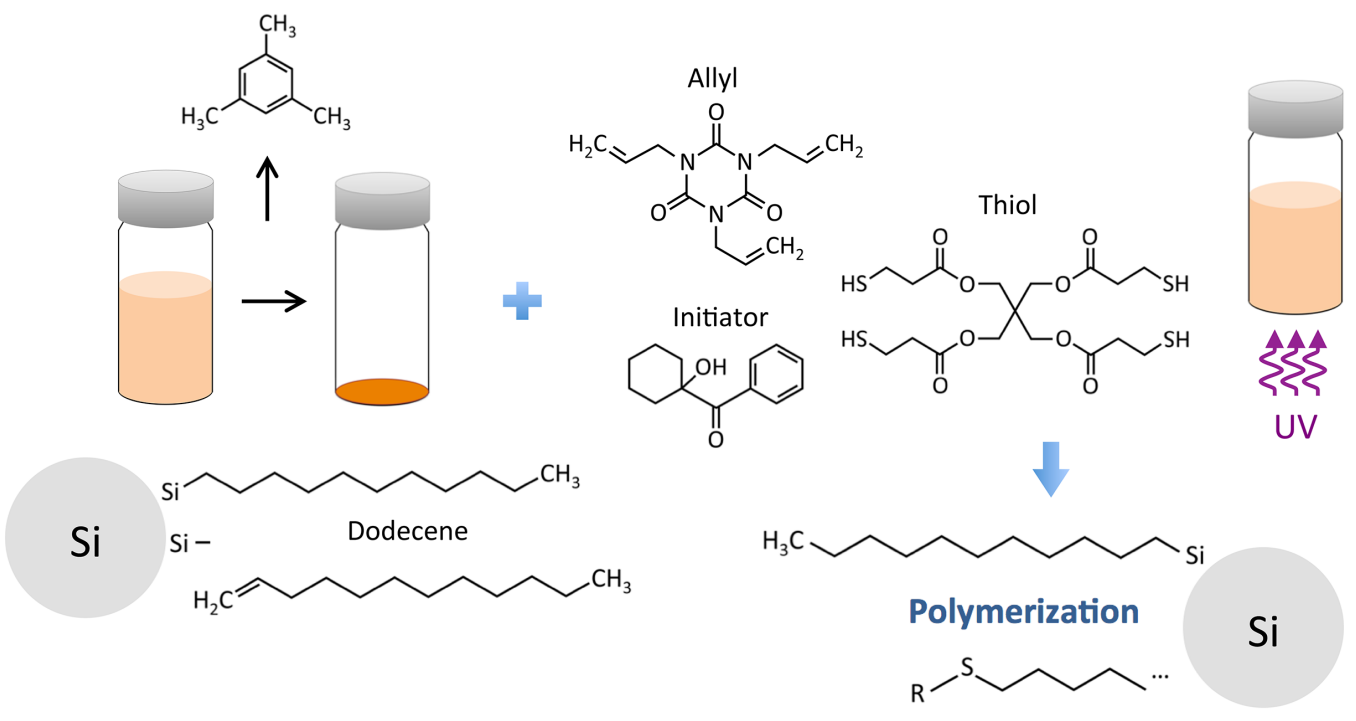

Scheme S1: Colloidal SiNCs passivated with 1-dodecene and suspended in mesitylene are dried under vacuum and the solvent is replaced with the OSTE resin mixture. Polymerization of the matrix and residual ligand is then activated by exposure to UV light. All samples were shielded from exposure to air and were processed in a nitrogen atmosphere. 

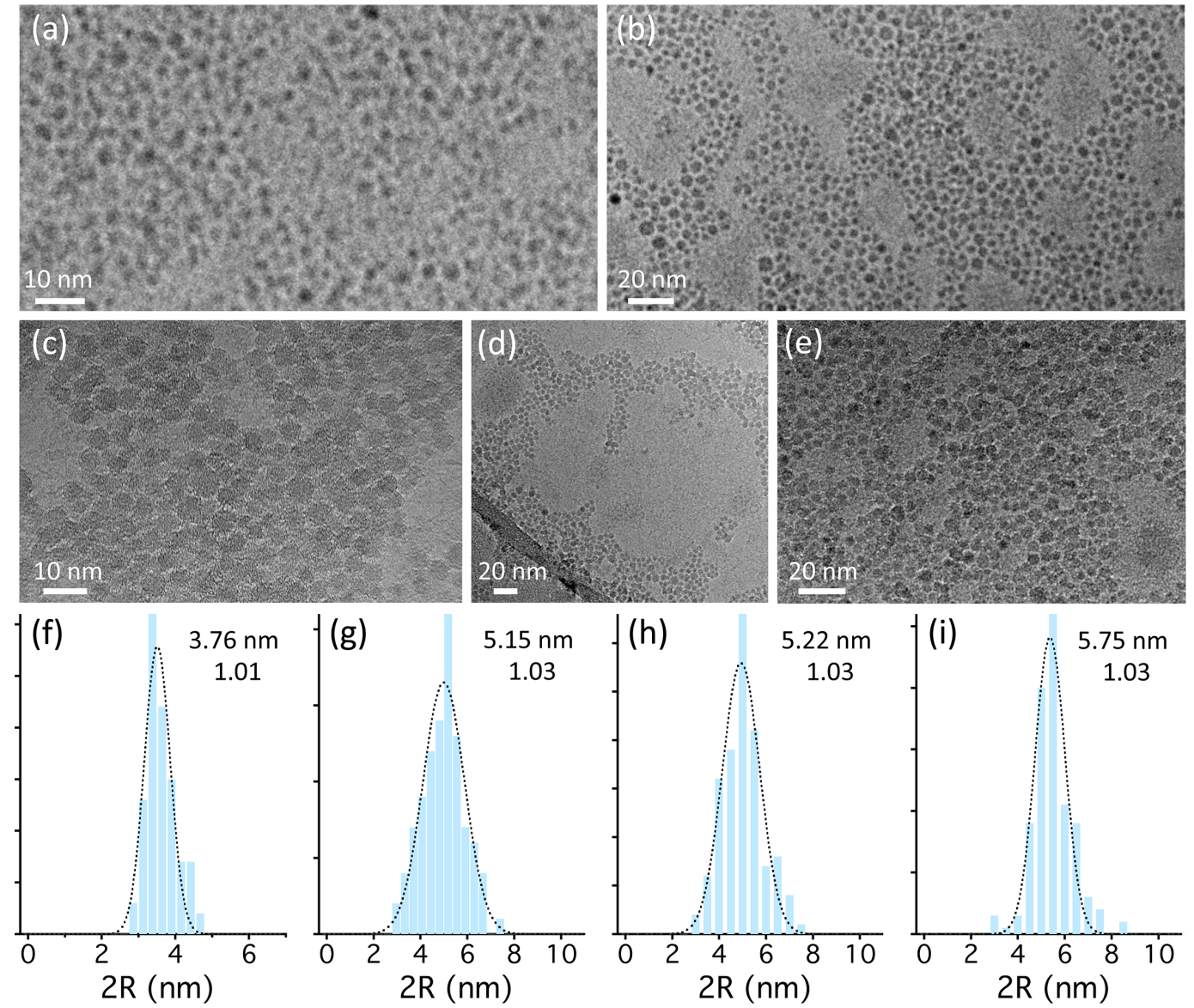

Figure S1. (a)-(e) Typical TEM image of SiNC colloids (PL peak near $750 \mathrm{~nm}, 875 \mathrm{~nm}$, $900 \mathrm{~nm}, 900 \mathrm{~nm}, 930 \mathrm{~nm}$, respectively) and size histograms based on TEM for samples with PL peaks near (f) $750 \mathrm{~nm}$, (g) $875 \mathrm{~nm}$, (h) $900 \mathrm{~nm}$, and (i) $930 \mathrm{~nm}$. Average size and polydispersity index are indicated next to each histogram. 

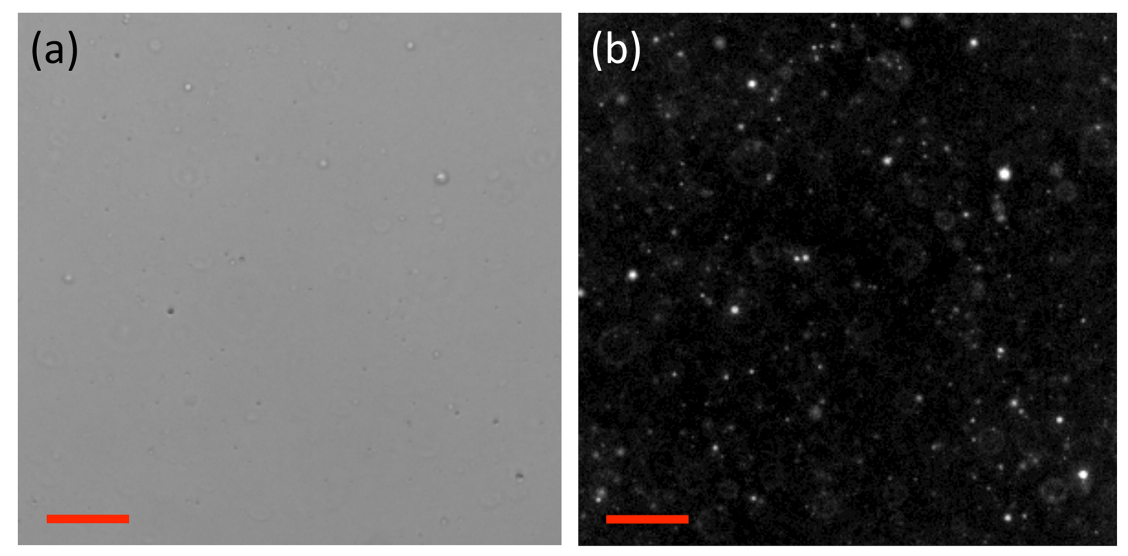

Figure S2. (a)-(b) Bright-field (left) and PL (right) microscopy images of a typical composite, where images were taken in transmission (left) and EPI illumination (right) on the via in which the composite was made. Scale bar is $50 \mu \mathrm{m}$.
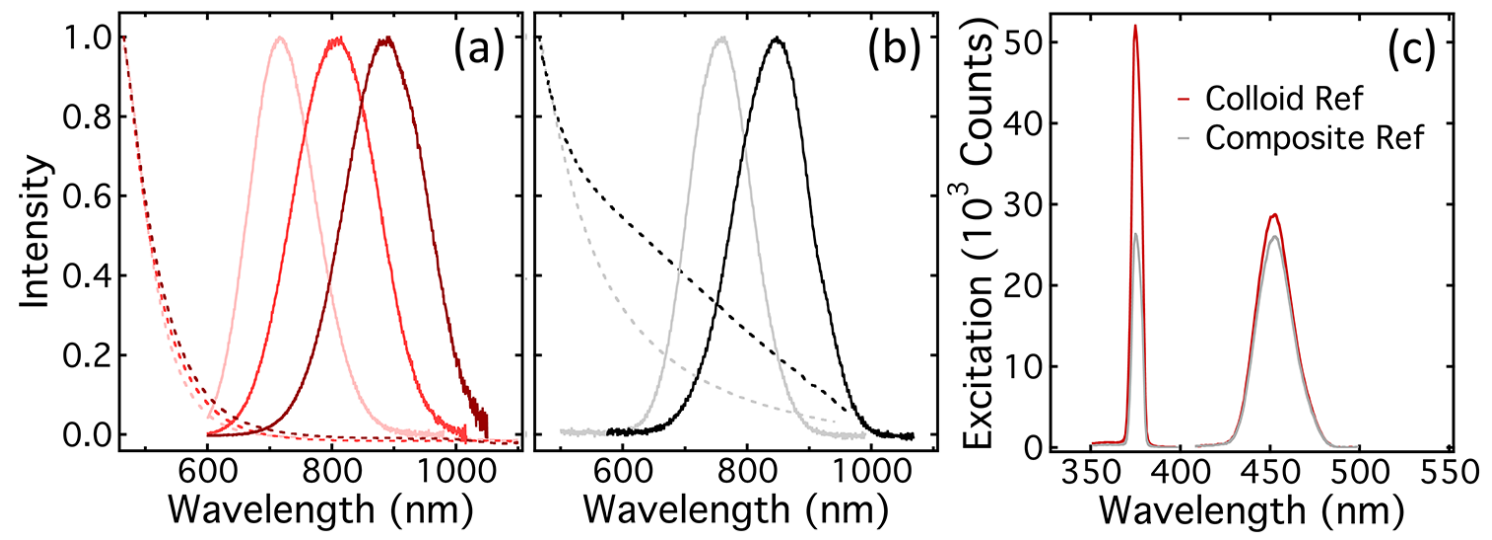

Figure S3. (a) Representative PL spectra (solid) and the corresponding absorption spectra (dashed) for colloidal SiNCs in mesitylene. PL spectra are normalized to 1 at the peak and absorption is normalized to 1 at $465 \mathrm{~nm}$. (b) A similar plot for SiNC composites, where the pure resin has been used as the reference for absorption measurements. (c) Comparison of the extinction between colloid and composite references (no SiNCs) for two optical inputs (375 nm laser and $450 \mathrm{~nm}$ LED). The composites show anomalously strong and variable absorption, which we attribute to the strong optical absorption of the pure resin at short wavelengths. 


\section{Bimodal PL Relaxation}

The analysis is taken from Refs. [53] and [55] of the main text. The decay is phenomenologically described by the bimodal stretched-exponential relaxation

$$
I(t)=I_{f} \exp \left[-\left(\frac{t}{\tau_{f}}\right)^{\alpha_{f}}\right]+I_{s} \exp \left[-\left(\frac{t}{\tau_{s}}\right)^{\alpha_{s}}\right]
$$

It is easiest to conceptualize a stretched exponential decay as a sum over a distribution of single-exponential decays, i.e.

$$
I_{i} \exp \left[-\left(\frac{t}{\tau_{i}}\right)^{\alpha_{i}}\right]=\sum_{j} \varphi_{i j} k_{j i_{\mathrm{R}}} e^{-k_{i j} t},
$$

where the subscript $j$ denotes a sub-channel of main channel $i(i=s$ or $f$ ). The quantity $\varphi_{i j}$ is the fraction of emitters in sub-channel $j, k_{i j}$ is the PL decay rate of sub-channel $j$, and $k_{i j_{\mathrm{R}}}$ is the associated radiative rate constant. The normalization is $\phi_{i}=\sum_{j} \varphi_{i j}$, where $\phi_{i}$ is the total fraction of emitters in each main channel, with $\phi_{s}+\phi_{f}=1$. At $t=0$, $I_{i}=\sum_{j} \varphi_{i j} k_{i j_{\mathrm{R}}}=\phi_{i}\left(\sum_{j} \varphi_{i j} k_{i j_{\mathrm{R}}} / \sum_{j} \varphi_{i j}\right)=\phi_{i}\left\langle k_{i_{\mathrm{R}}}\right\rangle=\phi_{i} / \tau_{i_{\mathrm{R}}}$, where $\tau_{i_{\mathrm{R}}}$ is radiative lifetime. The integrated intensity (QY) contains two terms: $\Phi=\Phi_{s}+\Phi_{f}$ with

$$
\Phi_{i}=I_{i} \int_{0}^{\infty} \exp \left[-\left(\frac{t}{\tau_{i}}\right)^{\alpha_{i}}\right] d t
$$

The above expression can be rewritten as

$$
\Phi_{i}=I_{i} \tau_{i} \frac{\Gamma\left(\alpha_{i}^{-1}\right)}{\alpha_{i}}=\phi_{i}\left(\frac{\tau_{i}}{\tau_{i_{\mathrm{R}}}}\right) \frac{\Gamma\left(\alpha_{i}^{-1}\right)}{\alpha_{i}}
$$

where $\Gamma(x)$ is the gamma function. The QY is

$$
\Phi=\Phi_{s}+\Phi_{f}=I_{s} \tau_{s} \frac{\Gamma\left(\alpha_{s}^{-1}\right)}{\alpha_{s}}+I_{f} \tau_{f} \frac{\Gamma\left(\alpha_{f}^{-1}\right)}{\alpha_{f}}
$$


For $\alpha_{i}=1$ (pure exponential relaxation) this simplifies to the usual expression:

$$
\Phi=I_{s} \tau_{s}+I_{f} \tau_{f}=\phi_{s}\left(\frac{\tau_{s}}{\tau_{s_{\mathrm{R}}}}\right)+\phi_{f}\left(\frac{\tau_{f}}{\tau_{f_{\mathrm{R}}}}\right) .
$$

For the normalization used here, $A_{s}=I_{s} /\left(I_{s}+I_{f}\right)$ and $A_{f}=I_{f} /\left(I_{s}+I_{f}\right)$, and the ratio $\Phi_{s} / \Phi_{f}$ can be expressed in terms of measured quantities as

$$
\Phi_{s} / \Phi_{f}=\left(\frac{A_{s}}{A_{f}}\right)\left(\frac{\tau_{s}}{\tau_{f}}\right)\left(\frac{\alpha_{f}}{\alpha_{s}}\right) \frac{\Gamma\left(\alpha_{s}^{-1}\right)}{\Gamma\left(\alpha_{f}^{-1}\right)}
$$

The slow mode dominates the QY (Fig. S5) and ignoring the contribution of the fast channel gives $\Phi \approx\left(\frac{\tau_{s}}{\tau_{s_{\mathrm{R}}}}\right) \frac{\Gamma\left(\alpha_{s}^{-1}\right)}{\alpha_{S}} \propto \tau_{s} / \tau_{S_{\mathrm{R}}}$.
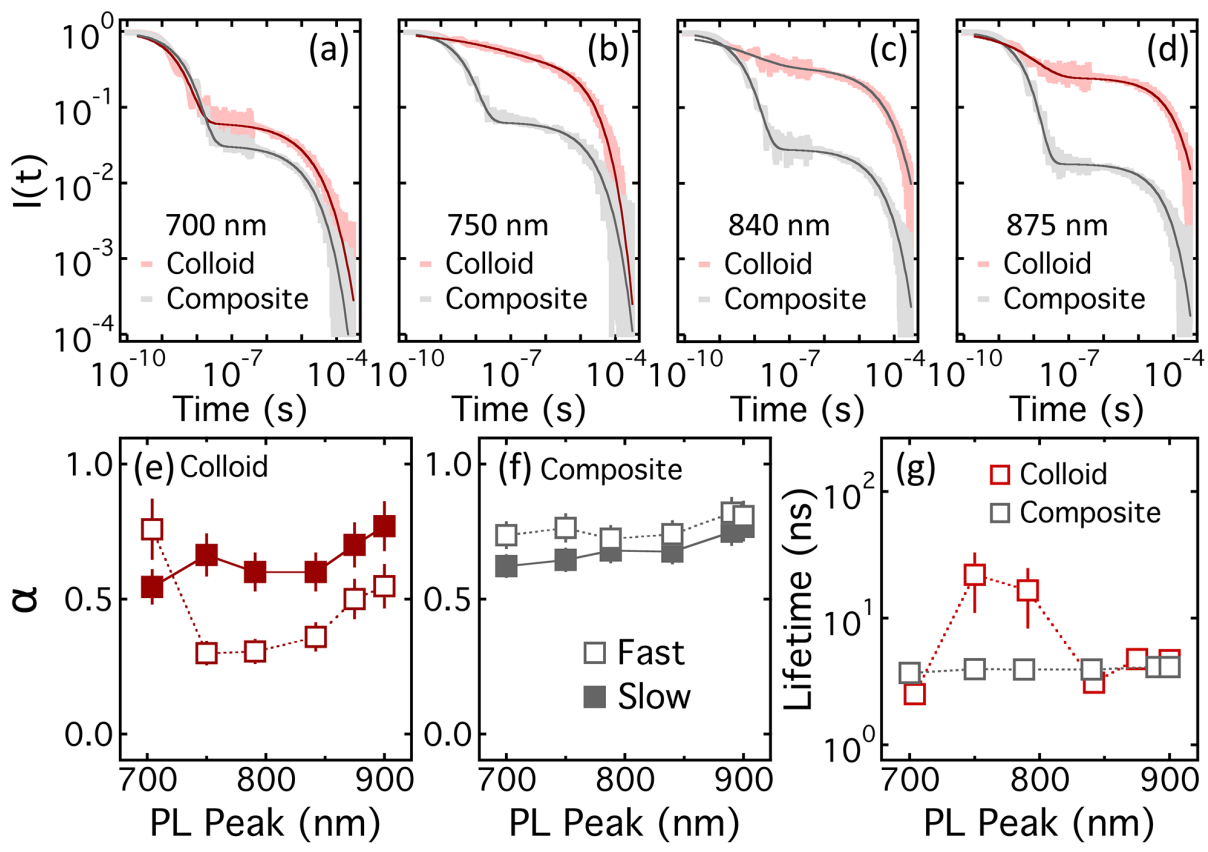

Figure S4. (a)-(d) Additional PL relaxation curves with the corresponding fits for the data in Fig. 2 of the main text. The PL peak of each sample is indicated. (e) Fast (open) and slow (closed) stretching exponents as a function of PL peak for the colloidal samples, and (f) a similar plot for the composite samples. (g) Fast lifetime vs. PL peak for colloids 
and composites. For the colloid, the increase in $\tau_{f}$ and the increase in stretching (small $\alpha_{f}$ ) near $800 \mathrm{~nm}$ correlate with the maximum in $A_{s}$ of the spectrally integrated intensity.
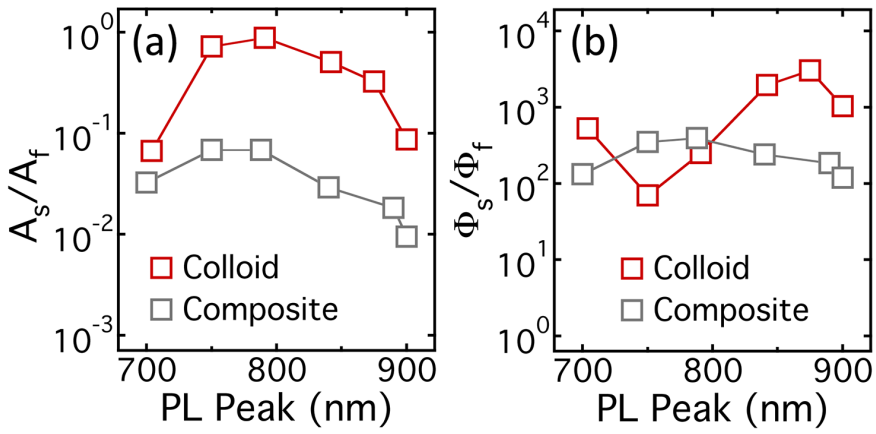

Figure S5. (a) The ratio $A_{s} / A_{f}$ as a function of PL peak, and (b) the corresponding implied ratio (Eq. S7) of the slow to fast contributions to the QY for colloidal (red) and composite (gray) samples represented in Fig. 2 of the main text.

\begin{tabular}{|c|c|c|c|c|c|c|c|c|c|}
\hline $\begin{array}{c}\text { Peak } \\
(\mathrm{nm})\end{array}$ & $\begin{array}{c}\text { QY } \\
( \pm 5 \%)\end{array}$ & $A_{s}$ & $A_{f}$ & $\begin{array}{c}\tau_{s} \\
(\mu \mathrm{s})\end{array}$ & $\begin{array}{c}\tau_{f} \\
(\mathrm{~ns})\end{array}$ & $\alpha_{s}$ & $\alpha_{f}$ & $\begin{array}{c}\tau_{s_{\mathrm{R}}} \\
(\mu \mathrm{s})\end{array}$ & $\begin{array}{c}2 R \\
(\mathrm{~nm})\end{array}$ \\
\hline 704 & 13.3 & $0.062 \pm$ & $0.938 \pm$ & 13.65 & $2.5 \pm$ & $0.54 \pm$ & $0.76 \pm$ & $180 \pm$ & - \\
& & 0.003 & 0.003 & \pm 1.4 & 0.2 & 0.02 & 0.11 & 35 & \\
\hline 750 & - & $0.42 \pm$ & $0.58 \pm$ & $14.7 \pm$ & $22 \pm$ & $0.66 \pm$ & $0.3 \pm$ & - & 3.76 \\
& & 0.02 & 0.02 & 1.5 & 10 & 0.02 & 0.05 & & $(1.01)$ \\
\hline 790 & 40.2 & $0.47 \pm$ & $0.53 \pm$ & $26.6 \pm$ & $16.6 \pm$ & $0.6 \pm$ & $0.31 \pm$ & $100 \pm$ & - \\
& & 0.02 & 0.02 & 2.7 & 8 & 0.02 & 0.05 & 20 & \\
\hline 842 & 45 & $0.34 \pm$ & $0.66 \pm$ & $36.3 \pm$ & $3.1 \pm$ & $0.6 \pm$ & $0.36 \pm$ & $120 \pm$ & - \\
& & 0.02 & 0.02 & 3.6 & 0.3 & 0.02 & 0.05 & 24 & \\
\hline 875 & 60.1 & $0.25 \pm$ & $0.75 \pm$ & $70 \pm$ & $4.8 \pm$ & $0.7 \pm$ & $0.5 \pm$ & $150 \pm$ & 5.15 \\
& & 0.01 & 0.01 & 7.0 & 0.5 & 0.02 & 0.08 & 30 & $(1.03)$ \\
\hline 900 & 32.0 & $0.08 \pm$ & $0.92 \pm$ & $82 \pm$ & $4.7 \pm$ & $0.77 \pm$ & $0.55 \pm$ & $300 \pm$ & 5.22 \\
& & 0.004 & 0.004 & 8.2 & 0.5 & 0.02 & 0.08 & 60 & $(1.03)$ \\
\hline
\end{tabular}

Table S1. Parameters for the PL relaxation of the colloidal samples presented in Fig. 2 of the main text, where QY is reported in \%. Diameter is based on TEM with polydispersity index indicated. 


\begin{tabular}{|c|c|c|c|c|c|c|c|c|c|}
\hline $\begin{array}{c}\text { Peak } \\
(\mathrm{nm})\end{array}$ & $\begin{array}{c}\text { QY* } \\
( \pm 5 \%)\end{array}$ & $A_{s}$ & $A_{f}$ & $\begin{array}{c}\tau_{s} \\
(\mu \mathrm{s})\end{array}$ & $\begin{array}{c}\tau_{f} \\
(\mathrm{~ns})\end{array}$ & $\alpha_{s}$ & $\alpha_{f}$ & $\begin{array}{c}\text { QY } \\
(\text { lifetime })\end{array}$ & $\begin{array}{c}2 R \\
(\mathrm{~nm})\end{array}$ \\
\hline 700 & 17.7 & $0.031 \pm$ & $0.969 \pm$ & $12.7 \pm$ & $3.7 \pm$ & 0.62 & $0.74 \pm$ & $11 \pm 3$ & - \\
& & 0.002 & 0.002 & 1.3 & 0.4 & \pm 0.02 & 0.05 & & \\
\hline 750 & - & $0.064 \pm$ & $0.936 \pm$ & $17.1 \pm$ & $3.9 \pm$ & $0.66 \pm$ & $0.76 \pm$ & - & 3.76 \\
& & 0.003 & 0.003 & 1.7 & 0.4 & 0.02 & 0.05 & & $(1.01)$ \\
\hline 788 & 51.2 & $0.064 \pm$ & $0.936 \pm$ & $21.3 \pm$ & $3.9 \pm$ & $0.6 \pm$ & $0.72 \pm$ & $32 \pm 8$ & - \\
& & 0.003 & 0.003 & 2.1 & 0.4 & 0.02 & 0.05 & & \\
\hline 840 & 47.9 & $0.028 \pm$ & $0.972 \pm$ & $29.7 \pm$ & $3.9 \pm$ & $0.6 \pm$ & $0.74 \pm$ & $37 \pm 10$ & - \\
& & 0.001 & 0.001 & 3.0 & 0.4 & 0.02 & 0.05 & & \\
\hline 890 & 61.7 & $0.018 \pm$ & $0.982 \pm$ & $39.3 \pm$ & $4.1 \pm$ & $0.7 \pm$ & $0.82 \pm$ & $34 \pm 9$ & 5.15 \\
& & 0.001 & 0.001 & 4.0 & 0.4 & 0.02 & 0.06 & & $(1.03)$ \\
\hline 900 & 51.4 & $0.009 \pm$ & $0.991 \pm$ & $50 \pm$ & $4.1 \pm$ & $0.77 \pm$ & $0.81 \pm$ & $20 \pm 5$ & 5.22 \\
& & 0.001 & 0.001 & 5.0 & 0.4 & 0.02 & 0.06 & & $(1.03)$ \\
\hline
\end{tabular}

Table S2. Parameters for the PL relaxation of the composite samples presented in Fig. 2 of the main text, where QY is reported in \%. *Indicates QY as measured in an integrating sphere.

\begin{tabular}{|c|c|c|c|c|c|c|c|c|}
\hline $\begin{array}{c}\text { Sample } \\
\text { (Fig. 2a) }\end{array}$ & $\begin{array}{c}\text { Peak } \\
(\mathrm{nm})\end{array}$ & $A_{s}$ & $A_{f}$ & $\begin{array}{c}\tau_{s} \\
(\mu \mathrm{s})\end{array}$ & $\begin{array}{c}\tau_{f} \\
(\mathrm{~ns})\end{array}$ & $\alpha_{s}$ & $\alpha_{f}$ & QY \\
\hline Colloid & 762 & $0.39 \pm$ & $0.61 \pm$ & 33.76 & 14.00 & $0.56 \pm$ & $0.31 \pm$ & $40 \pm 5$ \\
& & 0.02 & 0.02 & \pm 1.6 & \pm 5.0 & 0.02 & 0.11 & \\
\hline Dried & - & $0.33 \pm$ & $0.67 \pm$ & $8.2 \pm$ & $2.5 \pm$ & $0.40 \pm$ & $0.61 \pm$ & - \\
Colloid & & 0.02 & 0.02 & 1.0 & 0.5 & 0.02 & 0.05 & \\
\hline Composite & 767 & $0.08 \pm$ & $0.92 \pm$ & $32.5 \pm$ & $4.5 \pm$ & $0.55 \pm$ & $0.78 \pm$ & $33^{*}$ \\
& & 0.02 & 0.02 & 1.6 & 0.5 & 0.02 & 0.05 & $48 \pm 10$ \\
\hline
\end{tabular}

\begin{tabular}{|c|c|c|c|c|c|c|c|c|}
\hline $\begin{array}{c}\text { Sample } \\
\text { (Fig. 2b) }\end{array}$ & $\begin{array}{c}\text { Peak } \\
(\mathrm{nm})\end{array}$ & $A_{s}$ & $A_{f}$ & $\begin{array}{c}\tau_{s} \\
(\mu \mathrm{s})\end{array}$ & $\begin{array}{c}\tau_{f} \\
(\mathrm{~ns})\end{array}$ & $\alpha_{s}$ & $\alpha_{f}$ & $\mathrm{QY}$ \\
\hline Colloid & 815 & $0.47 \pm$ & $0.53 \pm$ & $97.7 \pm$ & $3.5 \pm$ & $0.71 \pm$ & $0.38 \pm$ & $70 \pm 5$ \\
& & 0.02 & 0.02 & 1.6 & 1.0 & 0.02 & 0.11 & \\
\hline Dried & - & $0.44 \pm$ & $0.56 \pm$ & $47.4 \pm$ & $2.15 \pm$ & $0.61 \pm$ & $0.35 \pm$ & - \\
Colloid & & 0.02 & 0.02 & 2.0 & 0.5 & 0.02 & 0.11 & \\
\hline Composite & 820 & $0.10 \pm$ & $0.90 \pm$ & $69.2 \pm$ & $4.5 \pm$ & $0.68 \pm$ & $0.72 \pm$ & $68^{*}$ \\
& & 0.02 & 0.02 & 2.5 & 0.5 & 0.02 & 0.05 & $75 \pm 10$ \\
\hline
\end{tabular}

Table S3. Parameters for the PL relaxation of the colloidal samples presented in Fig. 3 of the main text, where QY is reported in \%. Dried colloidal samples are exposed to air and hence will have a lower QY, which is apparent here as a shorter lifetime. *Indicates QY as measured in an integrating sphere. 


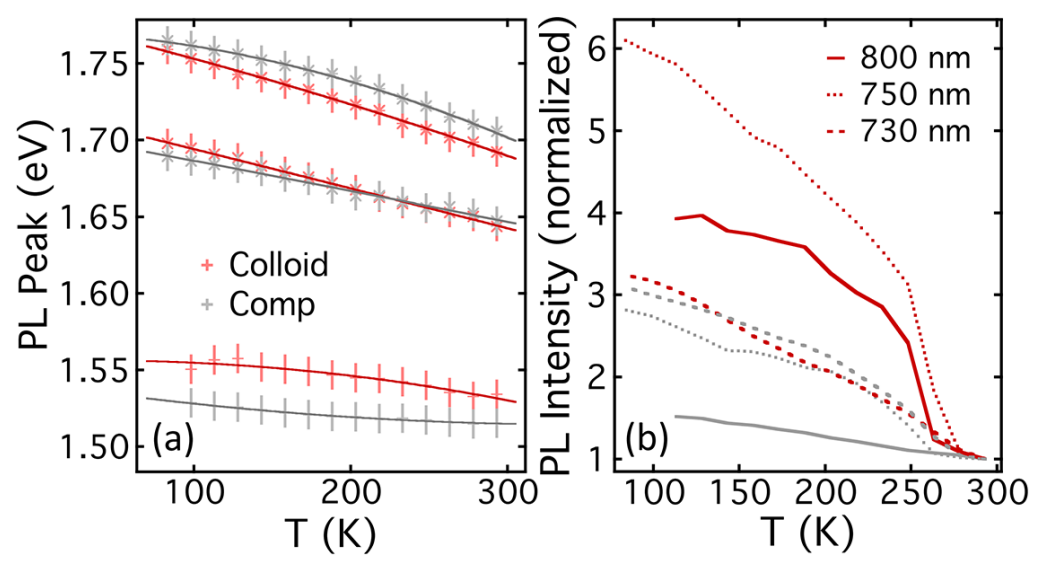

Figure S6. (a) Temperature dependent bandgap of three colloid/composite pairs with $2^{\text {nd }}$ order polynomial fits. (b) Temperature dependent PL intensity for the data in panel (a) normalized to unity at $25^{\circ} \mathrm{C}$. Colloidal data are red and composite data are gray.
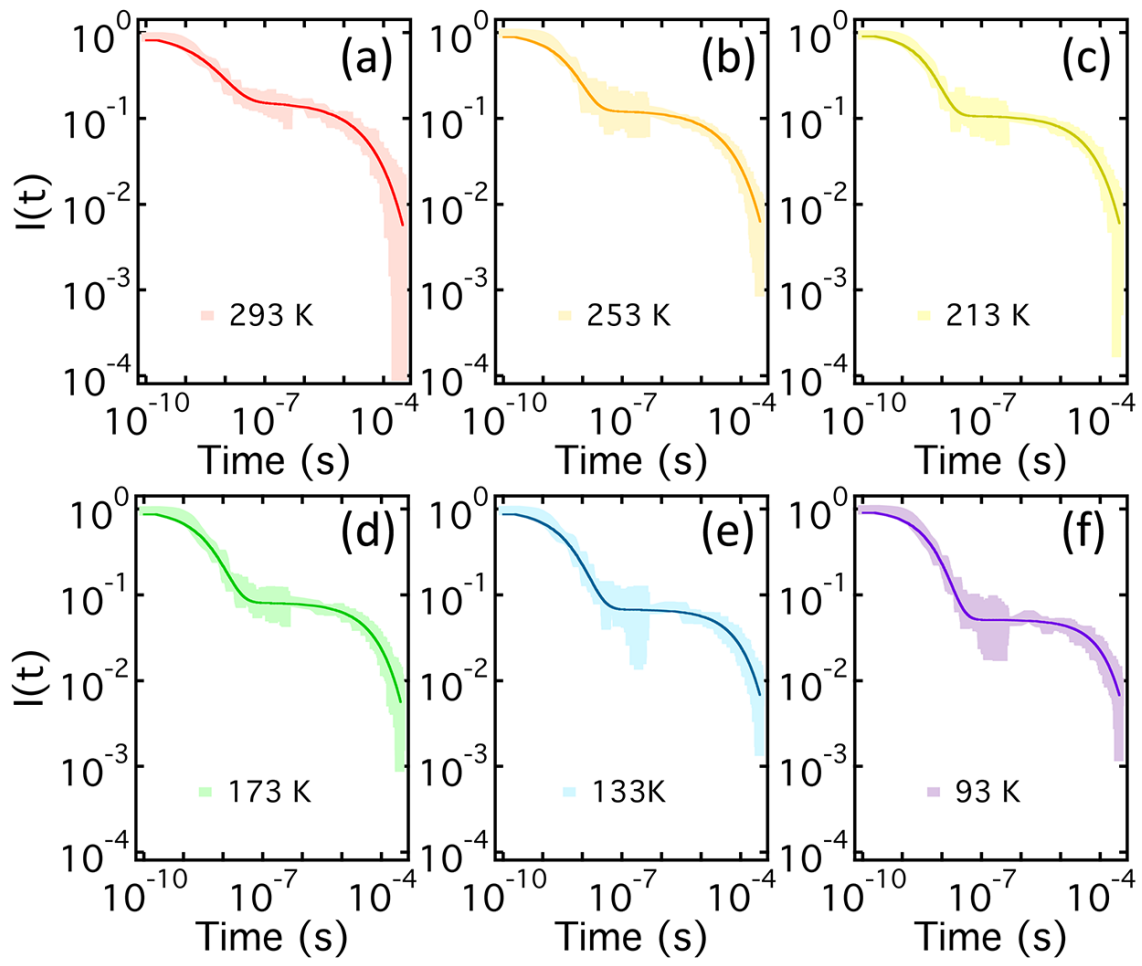

Figure S7. (a)-(f) Measured PL relaxation curves with fits for the temperature dependent composite detailed in the text. 


\begin{tabular}{|c|c|c|c|c|c|c|c|c|c|}
\hline$T(\mathrm{~K})$ & $\begin{array}{c}\text { PL Peak } \\
(\mathrm{nm})\end{array}$ & $A_{s}$ & $A_{f}$ & $\begin{array}{c}\tau_{s} \\
(\mu \mathrm{s})\end{array}$ & $\begin{array}{c}\tau_{f} \\
(\mathrm{~ns})\end{array}$ & $\alpha_{s}$ & $\alpha_{f}$ & $\begin{array}{c}\tau_{\mathrm{R}} \\
(\mu \mathrm{s})\end{array}$ & $\begin{array}{c}\text { QY } \\
(\text { lifetime })\end{array}$ \\
\hline 294 & 747 & $0.157 \pm$ & $0.843 \pm$ & $34.2 \pm$ & $3.0 \pm$ & 0.55 & $0.52 \pm$ & $130 \pm$ & $45 \pm 5$ \\
& & 0.005 & 0.005 & 1.3 & 0.4 & \pm 0.02 & 0.05 & 25 & \\
\hline 253 & 748 & $0.124 \pm$ & $0.876 \pm$ & $47.3 \pm$ & $3.7 \pm$ & $0.59 \pm$ & $0.68 \pm$ & 150 & 49.5 \\
& & 0.004 & 0.004 & 2.3 & 0.4 & 0.02 & 0.05 & & \\
\hline 213 & 744 & $0.108 \pm$ & $0.892 \pm$ & $58.5 \pm$ & $3.8 \pm$ & $0.65 \pm$ & $0.75 \pm$ & 157 & 48.5 \\
& & 0.003 & 0.003 & 2.0 & 0.4 & 0.02 & 0.05 & & \\
\hline 173 & 737 & $0.082 \pm$ & $0.918 \pm$ & $71.0 \pm$ & $3.6 \pm$ & $0.68 \pm$ & $0.64 \pm$ & 170 & 51 \\
& & 0.002 & 0.002 & 3.0 & 0.4 & 0.02 & 0.05 & & \\
\hline 133 & 728 & $0.068 \pm$ & $0.932 \pm$ & $98 \pm$ & $4.0 \pm$ & $0.74 \pm$ & $0.64 \pm$ & 211 & 56 \\
& & 0.002 & 0.002 & 4.0 & 0.4 & 0.02 & 0.06 & & \\
\hline 93 & 727 & $0.051 \pm$ & $0.949 \pm$ & $124 \pm$ & $4.9 \pm$ & $0.80 \pm$ & $0.72 \pm$ & 240 & 58.5 \\
& & 0.001 & 0.001 & 5.0 & 0.4 & 0.02 & 0.06 & & \\
\hline
\end{tabular}

Table S4. Parameters for fast and slow PL relaxation at each temperature detailed in

Figure S7 above. 